\title{
Determining the prevalence and density of asymptomatic Plasmodium falciparum infections in low and high transmission settings in Western Kenya
}

Christina Salgado ${ }^{1}$ Erik Gaskin ${ }^{2}$ Michael Macklin ${ }^{2}$ Eliud Onyango ${ }^{2}$ Rachel Surridge ${ }^{2}$ Srinivas Nallandhighal ${ }^{3}$ Jyoti Bhardwaj ${ }^{2}$ Dibyadyuti Datta ${ }^{2}$ Lindsey Turnbull ${ }^{2}$ Wendy P O'Meara ${ }^{4,5}$ George Ayodo ${ }^{6,7}$ Chandy C John ${ }^{2}$ Tuan M Tran ${ }^{2}$

${ }^{1}$ Indiana University School of Medicine; ${ }^{2}$ Indiana University School of Medicine, Department of Infectious Disease; ${ }^{3}$ Michigan Medicine, Department of Urology ; ${ }^{4}$ Duke University, Duke Global Health Institute; ${ }^{5}$ Duke University School of Medicine, Division of Infectious Disease; ${ }^{6}$ Kenya Medical Research Institute, Center for Global Health Research; ${ }^{7}$ Jaramogi Oginga Odinga University of Science and Technology

Background/Objective: Malaria remains a global health burden as 2018 saw 228 million cases worldwide. Pre-elimination efforts aim to decrease disease transmission, which depends on development of asexual parasites to sexual gametocytes within the human host. To better determine the relationship between asexual parasites and gametocytes, we aim to quantify both stages within the same blood sample by developing a multiplex RT-qPCR assay. We hypothesize that gametocytemia decreases linearly with asexual parasitemia at higher parasitemias but increases at lower parasitemia to improve transmission probability.

Methods: We conducted a cross-sectional survey of healthy participants aged 0 to 92 years at four sites in Western Kenya (Ajigo, Webuye, Kipsamoite, Kapsisywa) with varying levels of transmission to determine prevalence of asymptomatic $P$. falciparum blood-stage infections. Parasite density was quantified by light microscopy and by quantitative PCR of genomic DNA isolated from dried blood spots using varATS primers. For varATS-positive samples, we isolated RNA from whole-blood and performed stage-specific quantification by multiplex Taqman RTqPCR targeting PfPIESP2 (asexual), Pfs25 (female), and Pfs230p (male).

Results: Among all participants, 127 of 1355 (9.37\%) were positive for asymptomatic $P$. falciparum infection by varATS-qPCR. Using varATS positivity as the standard, multiplex RTqPCR targeting PfPIESP2 yielded a sensitivity of $78 \%$. Across sites, overall parasite prevalence and gametocyte prevalence were highest in Ajigo (42.1\%; 36\%) followed by Webuye (10.5\%; 9\%) and Kipsamoite/Kapsisywa (0.873\%; 0.1\%). Pfs25-quantified gametocytemia decreased with decreasing varATS-quantified asexual parasitemia; however, the data poorly fit a linear model $\left(R^{2}=0.088\right)$.

Conclusions: PfPIESP2 detected $78 \%$ of varATS-positive samples, suggesting it may be poorly sensitive at detecting low-density blood-stage infections. Assay design may improve with a more sensitive gene target and further optimization. Based on quantification results, the 
relationship between gametocytemia and asexual parasitemia appears direct but non-linear.

Further work is necessary to better model the relationship between stage-specific parasitemia levels. 